Anestesiología

Abril-Junio 2022

Vol. 45. No. 2. pp 114-120

doi: 10.35366/103886

\section{Abordaje perioperatorio en cirugía neurológica con el paciente despierto}

\section{Perioperative approach in neurological surgery with the patient awake}

\author{
Acad. Dr. Eduardo Homero Ramírez-Segura,* Dra. Bianca Yannet Bataz-Pita ${ }^{\ddagger}$
}

Citar como: Ramírez-Segura EH, Bataz-Pita BY. Abordaje perioperatorio en cirugía neurológica con el paciente despierto. Rev Mex Anestesiol. 2022; 45 (2): 114-120. https://dx.doi.org/10.35366/103886

RESUMEN. La craneotomía con el paciente despierto se refiere a aquellos procedimientos en los que el paciente conserva su estado de consciencia durante toda la cirugía o en parte de ésta con el objetivo de explorar la integridad de sus funciones cerebrales superiores en tiempo real. Estas técnicas neuroanestésicas son útiles para ayudar al neurocirujano a preservar la integridad del tejido cerebral, o bien, no causar mayor daño del que la propia enfermedad ha causado.

ABSTRACT. Awake craniotomy refers to those procedures in which the patient remains conscious for all or part the time, with the aim of explore in real time the integrity of their higher brain functions. This kind of neuroanesthetic techniques are useful in assisting the neurosurgeon to preserve the integrity of the brain or not to damage more than what the disease has caused.

Considerando los factores favorables hará viable su plan; considerando los factores desfavorables, quizá logre resolver las dificultades.

El arte de la guerra

Sun Tzu

\section{INTRODUCCIÓN}

T a craneotomía con paciente despierto (CPD) se refiere habitualmente a cualquier procedi$\checkmark$ miento neuroquirúrgico intracraneal, en el cual el paciente conserva el estado de consciencia durante todo el evento o en alguna parte de éste, así preservando la capacidad de responder de manera activa ante los estímulos provocados por parte del equipo médico.

De primera instancia, la cirugía neurológica de CPD se diseñó para pacientes sometidos a cirugía neurológica funcional para el tratamiento de la epilepsia, no obstante, con el avance y perfeccionamiento en las técnicas quirúrgicas, anestésicas, monitoreo neurofisiológico y de neuroimagen, en la actualidad se realiza cirugía neurológica con paciente despierto en patologías que involucran áreas elocuentes y/o motoras, así como en aquéllos sometidos a cirugía estereotáctica funcional mediante la implantación de estimuladores cerebrales profundos (DBS, por sus siglas en inglés Deep Brain Stimulation) (Figura 1). La cirugía neurológica con paciente despierto se ha practicado desde años remotos; el primer reporte publicado data de 1886 en Inglaterra, Sir Victor Horsley (pionero de la cirugía neurológica, 1857-1916) resecó una lesión epileptógena en un hombre de 22 años, en la cual el paciente fue despertado durante la cirugía ${ }^{(1)}$. Penfield (autor del homúnculo cortical, «homúnculo de Penfield», 1891-1976) proponía que los pacientes con patología neurológica funcional deberían ser operados despiertos y realizando alguna actividad compleja como leer o tocar un instrumento, por mencionar algunas ${ }^{(2,3)}$.

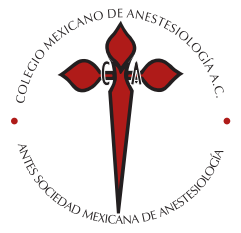

Palabras clave: Craneotomía despierto, neuroanestesia, resección tumoral cerebral, estimulador cerebral profundo.

Keywords: Awake craniotomy, neuroanesthesia, brain tumor resection, deep brain stimulation.

* Médico Naval, Anestesiólogo y Neuroanestesiólogo, Académico de Número-Academia Mexicana de Cirugía. Gpo. Neuroquirúrgico Nave Medical, Unidad Gamma Knife - Fundación Clínica Médica Sur. Ex Director de la Escuela de Posgrados en Sanidad NavalUniversidad Naval, Ex Jefe de División de Cirugía y Coordinador de Quirófanos, Hospital Naval de Alta Especialidad, Secretaría de Marina - Armada de México. Revisor Editorial Revista Mexicana de Anestesiología, Colegio Mexicano de Anestesiología, A.C.; Revista Cirugía y Cirujanos - Academia Mexicana de Cirugía; Archivos de Neurociencias, Instituto Nacional de Neurología y Neurocirugía.

‡ Médico Naval, Anestesióloga y Neuroanestesióloga. Coordinadora de Residentes.

Escuela de Postgrados en Sanidad Naval, Universidad Naval. Secretaría de Marina, Armada de México. México.

Correspondencia:

Acad. Dr. Eduardo Homero Ramírez-Segura

E-mail: ehramseg@gmail.com

Recibido: 28-04-2021

Aceptado: 05-07-2021 
Esta técnica anestésica se ha vuelto un procedimiento cada vez más utilizado para resección de lesiones cerebrales en áreas elocuentes y/o motoras, no dudemos que en un futuro se extienda a cualquier área del cerebro y a otros procedimientos intracraneales de forma rutinaria, como la descompresión microvascular, para valorar en tiempo real qué tanto disminuye el dolor al momento de la liberación nerviosa ${ }^{(4)}$.

El objetivo fundamental de esta técnica anestésica es brindar las herramientas al neurocirujano, para que lleve a cabo la resección de la lesión o el abordaje del blanco anatómico target con mayor seguridad y preservación al máximo de las áreas anatómicas (elocuentes y/o motoras), así como conservar la integridad del paciente, o bien no aumentar el déficit neurológico ya provocado por la propia enfermedad ${ }^{(2)}$. El manejo anestésico de este tipo de pacientes es un desafío para el neuroanestesiólogo, ya que se requiere de una función cerebral totalmente preservada para un óptimo monitoreo en tiempo real de las funciones cerebrales superiores y/o motoras (Figura 2), lo cual es posible gracias a los avances en la neurofarmacología, formación del anestesiólogo y al trabajo en equipo ${ }^{(5,6)}$. Existen reportes de casos en los que citados beneficios se han otorgado a pacientes pediátricos ${ }^{(7)}$, así como en mujeres embarazadas $^{(8)}$, es un procedimiento seguro en ambos casos. En el Instituto Nacional de Pediatría (México) el grupo de trabajo de la Dra. León Álvarez (neuroanestesióloga) realiza CPD en población pediátrica ${ }^{(9-11)}$. Aunado a todo lo anterior, es un procedimiento con mejor rentabilidad, ya que reduce los costos hospitalarios al disminuir el consumo de fármacos, acortar la estancia en unidades de cuidados intensivos e intrahospitalaria ${ }^{(12,13)}$.

La mayoría de la evidencia está basada en estudios retrospectivos o series de casos, en donde los pacientes fueron reportados con tumores cerebrales de bajo grado según la Organización Mundial de la Salud; sin embargo, ha ido en aumento el empleo de esta técnica para tumores de alto grado, mostrando de igual manera un mejor pronóstico, por lo que en la actualidad se están realizando ensayos clínicos aleatorizados, como el estudio The SAFE-trial: safe surgery for glioblastoma multiforme: awake craniotomy versus surgery under general anesthesia ${ }^{(14)}$, con lo que se pretende esclarecer si la técnica podría ser el procedimiento ideal para tumores de alto grado como lo es el glioblastoma ${ }^{(14)}$.

\section{VALORACIÓN PREANESTÉSICA Y PREOPERATORIA}

Para determinar si un paciente es candidato a cirugía neurológica bajo CPD, es indispensable contar con un equipo multidisciplinario (Figura 3) que «hable el mismo idioma»(2,15,16), ya que todos los participantes deben estar familiarizados con la técnica, de preferencia tener formación en neurociencias y tener una comunicación efectiva para que cada integrante cumpla con las funciones en las diferentes etapas y conozcan

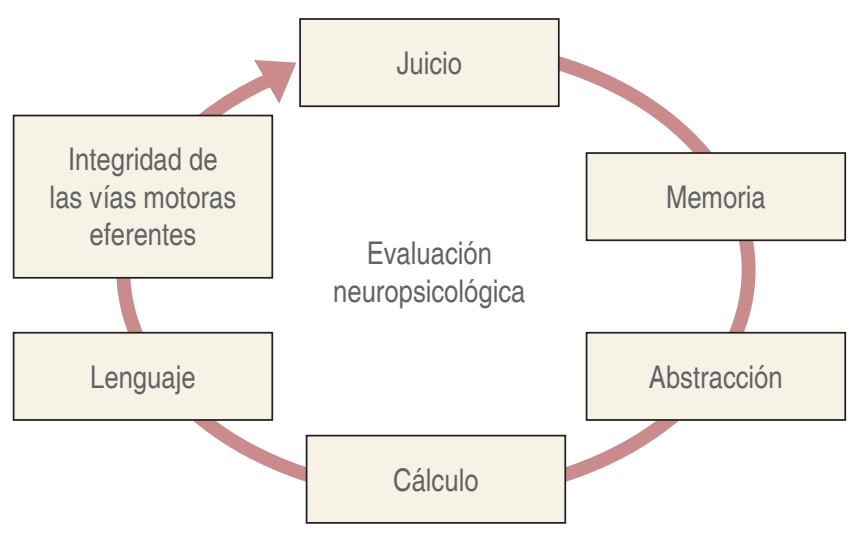

Figura 2: Valoración neurológica en tiempo real en craneotomía con paciente despierto (CPD).
Figura 1:

Implante de estimulación cerebral profunda (DBS, por sus siglas en inglés); paciente despierto.
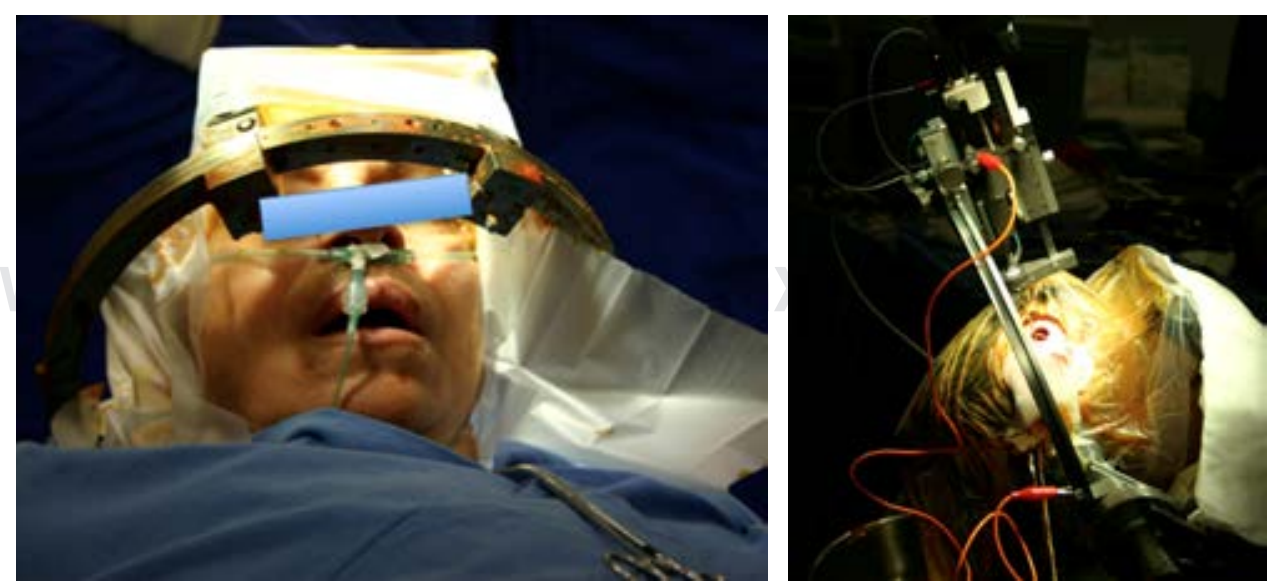


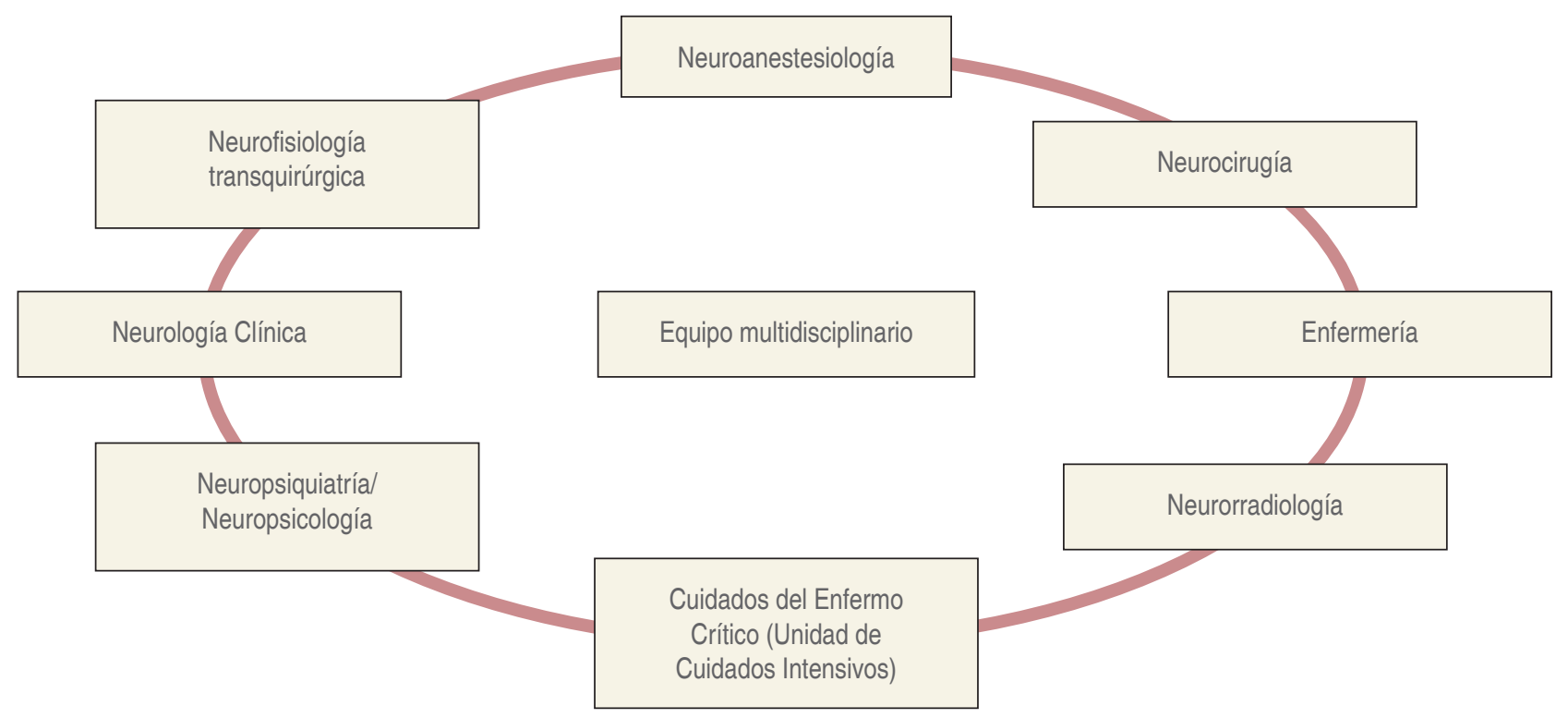

Figura 3: Equipo multidisciplinario en neurociencias.

\begin{tabular}{|c|c|c|c|}
\hline \multicolumn{4}{|c|}{ Técnica anestésica } \\
\hline Etapa & Dormido & Despierto & Dormido \\
\hline Procedimiento quirúrgico & $\begin{array}{l}\text { - Monitoreo invasivo } \\
\text { - Craneotomía }\end{array}$ & $\begin{array}{l}\text { - Trépano o craneotomía } \\
\text { - Mapeo } \\
\text { - Resección de lesión o colocación de } \\
\text { implante de dispositivo cerebral }\end{array}$ & $\begin{array}{l}\text { - Hemostasia } \\
\text { - Cierre craneotomía } \\
\text { - Implante generador torácico }\end{array}$ \\
\hline \multicolumn{4}{|c|}{ Modalidad } \\
\hline Despierto & & CAM & \\
\hline DD & & Bloqueo de escalpe + sedación & TIVA o AGB \\
\hline DDD & Bloqueo de escalpe + TIVA o AGB & Sedación & TIVA o AGB \\
\hline
\end{tabular}

$\mathrm{DD}=$ despierto-dormido; $\mathrm{DDD}$ = dormido-despierto-dormido; $\mathrm{AGB}$ = anestesia general balanceada; $\mathrm{TIVA}$ = anestesia total intravenosa; $\mathrm{CAM}$ = cuidados anestésicos monitorizados.

las posibles complicaciones a ocurrir, y en caso de detectar alguna, actuar de forma certera e inmediata. Asimismo, la valoración neuropsicológica es fundamental para considerar si el paciente es apto para ser manejado con esta técnica ${ }^{(2)}$.

La única contraindicación absoluta para realizar CPD es el rechazo del paciente o sus familiares. Aun así, el neuroanestesiólogo debe poner en duda la viabilidad del procedimiento cuando en la valoración preanestésica encuentre contraindicaciones relativas como: predictores de vía aérea difícil, existencia de una barrera del lenguaje (apoyado por la valoración neuropsicológica), tos incoercible, apnea obs- tructiva del sueño, obesidad mórbida y alteraciones psicológicas que condicionen incapacidad para mantenerse quieto durante el procedimiento en la fase de despierto, ya que la colaboración del caso durante la etapa de vigilia es crucial para el éxito de la cirugía; en la valoración preanestésica el neuroanestesiólogo debe explicar detalladamente las etapas de la cirugía y establecer una adecuada relación médicopaciente con el fin de disminuir la ansiedad ${ }^{(17)}$, incluso en el contexto de pacientes psiquiátricos se ha demostrado que la visita preanestésica es fundamental para garantizar el proceder de la técnica ${ }^{(18)}$. 


\section{PLANEAMIENTO DE LA TÉCNICA ANESTÉSICA}

Los criterios para llevar a cabo la técnica anestésica para cirugía neurológica con CPD pueden variar interinstitucionalmente e incluso en un mismo centro hospitalario, dependiendo de la «escuela de formación», de la experiencia del neuroanestesiólogo y del neurocirujano, los recursos hospitalarios (humanos, tecnológicos, infraestructura y económicos), así como de las condiciones clínicas en particular de cada paciente ${ }^{(19)}$.

Existen tres modalidades anestésicas en torno a este tipo de cirugía (Tabla 1):

1. Paciente despierto durante todo el procedimiento (en nuestro grupo de trabajo no lo practicamos).

2. Despierto-dormido (DD) (awake-asleep). Despierto desde el inicio y hasta el momento de la resección tumoral y/o la implantación de los DBS, y una vez llevado a cabo el procedimiento, «se duerme» al paciente para el cierre y/o la «tunelización» de los cables y la implantación del generador en la región infraclavicular; despertándolo al término del procedimiento.

3. Dormido-despierto-dormido (DDD) (asleep-awakeasleep). Dormido desde el inicio, se monitoriza e «invade» (intubación, colocación de catéteres venoso y arterial, sonda urinaria, etcétera), se lleva a cabo la craneotomía y una vez realizada ésta, se «despierta» para llevar a cabo la resección tumoral con valoración neurológica en tiempo real; hecha la resección de la lesión, se procede a «dormir» al paciente de nuevo para cerrar la craneotomía y despertarlo al término del procedimiento ${ }^{(5)}$.

Como se mencionó, la elección de la técnica anestésica es multifactorial, pero las metas siempre serán las mismas: en la etapa de dormido las de una anestesia general (adecuado estado anestésico, estabilidad hemodinámica, adecuada perfusión cerebral, etceétra); en la etapa de despierto, garantizar la oxigenación, estabilidad hemodinámica, confortabilidad, adecuada analgesia (en nuestro grupo de trabajo, realizamos bloqueo regional de escalpe ${ }^{(2)}$ en cualesquiera de las dos técnicas DD o DDD) y sedación que permita la integridad de las funciones cerebrales superiores, así como una adecuada respuesta motora durante el mapeo (Figura 4)(20).

\section{MANEJO DE VÍA AÉREA}

Una de las interrogativas, retos e inquietudes del anestesiólogo en estas técnicas anestésicas es determinar el tipo de dispositivo que debe utilizarse para protección y manejo de la de
Figura 4:

Mapeo y estimulación cerebral.

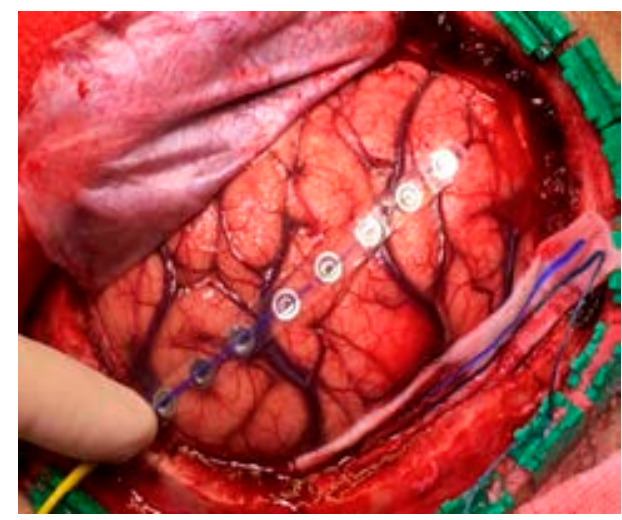

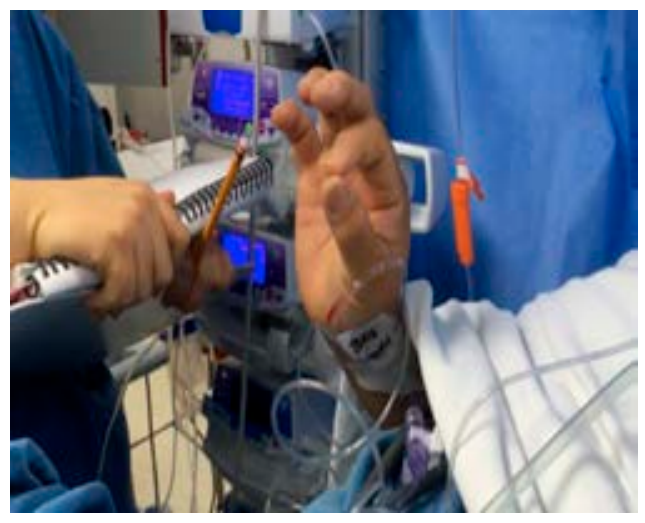
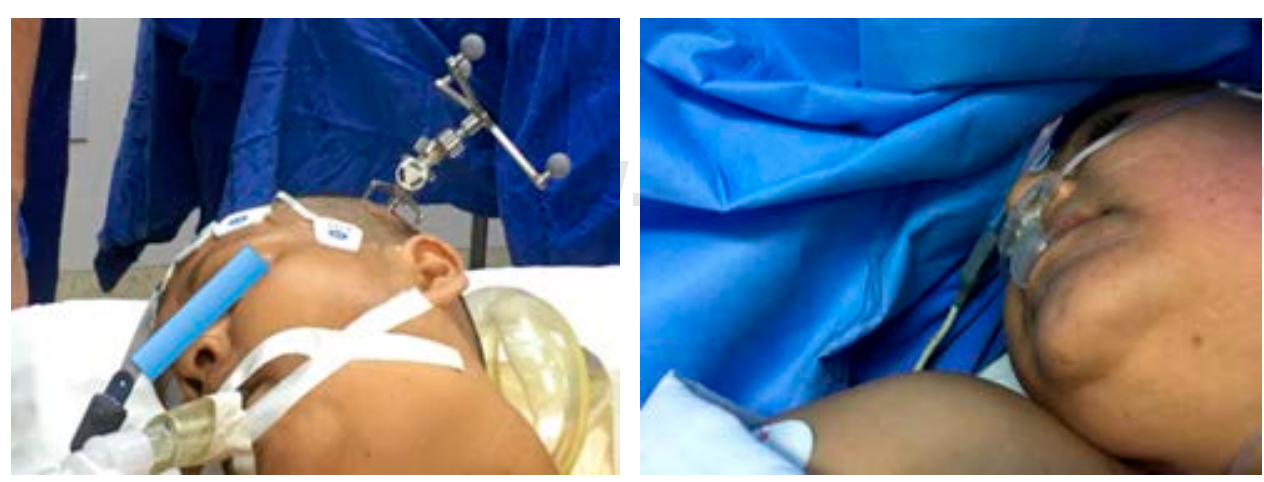

Figura 5:

Manejo de la vía aérea con mascarilla laríngea. 
vía aérea durante la etapa de dormido (tubo endotraqueal vs mascarilla laríngea) (Figura 5). Si bien la cánula endotraqueal con neumotaponamiento garantiza la entrada y salida del volumen corriente, así como protección completa de la vía aérea, puede ser mucho más molesto para el paciente, con mayor riesgo de presentar tos al momento de irlo emergiendo del plano anestésico, lo que representa la posibilidad de provocar aumento de la presión intracraneal con una consecuente hernia extracraneal (Figura 6). Incluso hay «escuelas o corrientes» que recomiendan no extubar en la fase de despierto en casos en los que no se vaya a explorar lenguaje, no obstante, por lo referido en líneas previas, se requiere de mucha experiencia para mantenerlo en un adecuado plano anestésico para que no tosa (intubado), pero que esté en capacidad de realizar las actividades motoras que se le soliciten ${ }^{(2)}$. Por el contrario, la mascarilla laríngea ha demostrado ser una alternativa segura para estos procedimientos, aunque no debemos olvidar que la posición «semilateral» del paciente puede condicionar fugas de la vía aérea y dificultar el reposicionamiento en el segundo tiempo de dormido ${ }^{(21,22)}$.

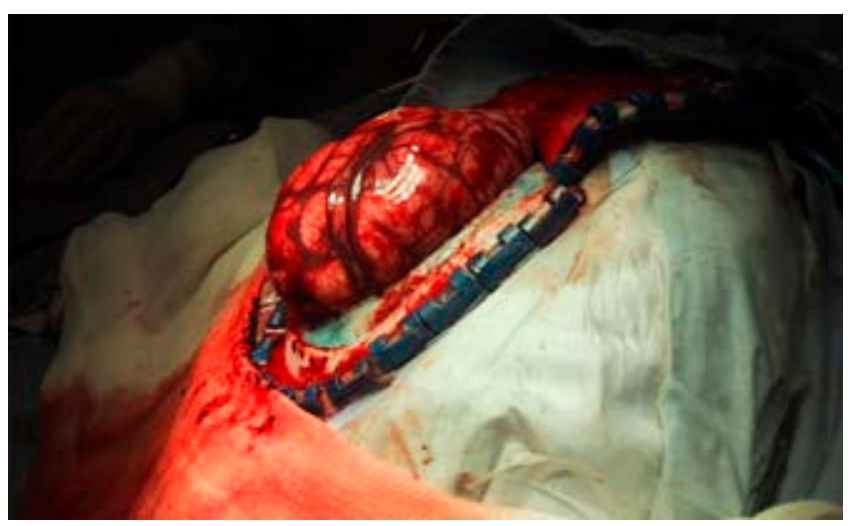

Figura 6: Hernia extracraneal por aumento de la presión intracraneal.
Para el manejo anestésico, se prefieren aquellos fármacos con un tiempo rápido de inicio de acción y la mayor estabilidad hemodinámica posible; el régimen propofolremifentanilo es recomendable para todas las fases de la cirugía, no obstante, para la etapa de vigilia se han estudiado otras combinaciones como propofol-dexmedetomidina, considerándola tan efectiva como propofol-remifentanilo pero con menos efectos secundarios para la sedación consciente durante la $\mathrm{CPD}^{(23)}$.

La anestesia total intravenosa, de preferencia TCI (del inglés Target Controlled Infusion), es una buena alternativa, ya que de algún modo es «programable y predecible» el despertar (Figura 7). Los modelos farmacocinéticos del propofol se han estudiado ampliamente, el modelo de Schneider mostró un mayor acercamiento a las concentraciones plasmáticas vs el modelo de Marsh. La recuperación de la consciencia en Schneider se presenta a una CPD de 1.2 $\pm 0.4 \mu \mathrm{g} / \mathrm{mL}$ con un BIS de $77 \pm 7$. Y el comienzo de las pruebas neurológicas es posible con una medición de BIS de $90 \pm 6$. Nuestra experiencia coincide con lo reportado en la literatura médica internacional, con valores de 81-82 de BIS obtenemos adecuada respuesta en la valoración neuropsicológica y motora, podemos observar en la Figura 8 como van aumentando gradualmente los valores de BIS hasta la fase de despierto ${ }^{(24)}$.

\section{COMPLICACIONES}

Las complicaciones de esta modalidad anestésica pueden ser multifactoriales; asociadas a una mala elección del paciente, relacionadas directamente a una mala técnica anestésica, como pudiera ser una sedación insuficiente que lleve a «abortar» el procedimiento por agitación o ansiedad, o bien, una sedación muy profunda que impida realizar la exploración neurológica en tiempo real, así
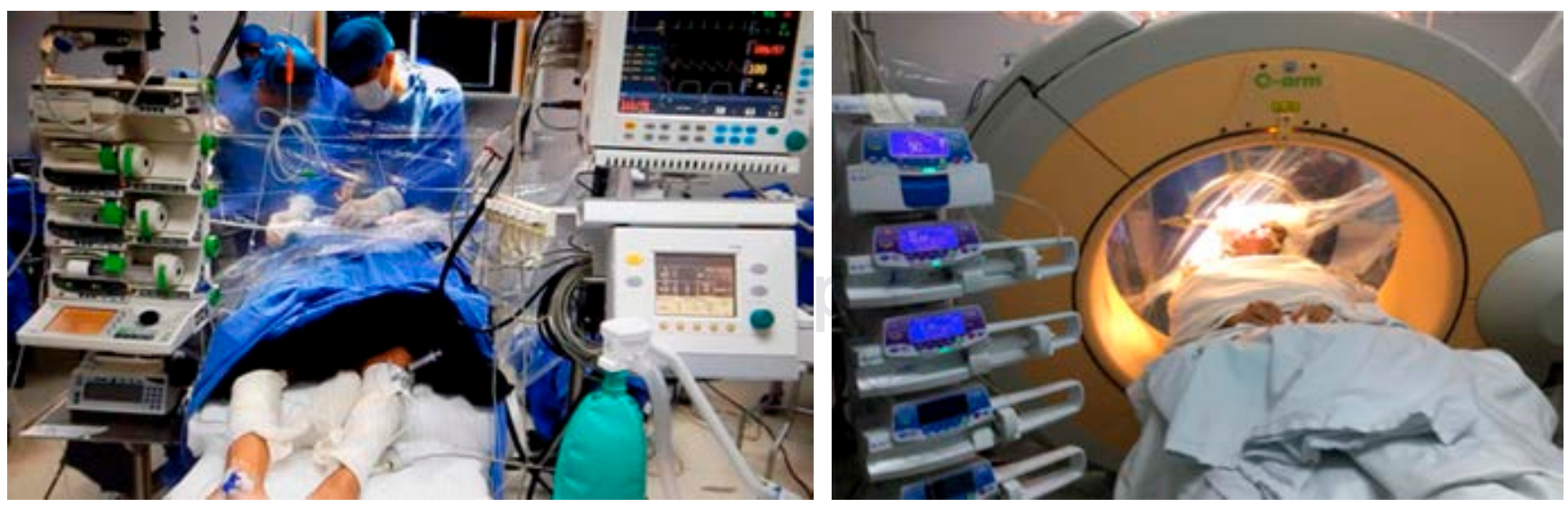

Figura 7: Perfusores de infusión controlada a objetivo (TCI). 


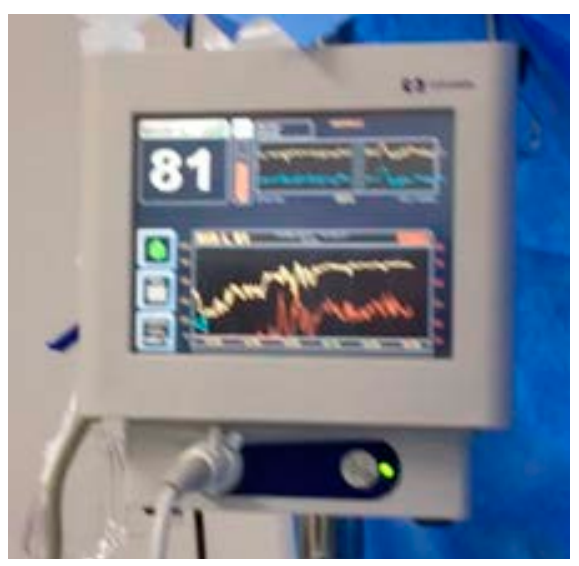

Figura 8:

Valores de BIS en la fase de despierto.

como contingencias asociadas a un mal manejo de la vía aérea $^{(25)}$, edema cerebral, crisis convulsivas mal manejadas, entre otras. A pesar de los estudios reportados, podemos decir que no existen factores contundentes que puedan predecir la aparición de convulsiones durante el tiempo del mapeo ${ }^{(25,26)}$.

\section{CONCLUSIONES}

Esta técnica anestésica es una herramienta más para que el neurocirujano pueda llevar a cabo la cirugía neurológica intracraneal con mayor seguridad, sin provocar mayor daño que el ya generado por la propia enfermedad. En general, la práctica de la anestesiología requiere de sólidos conocimientos teóricos, habilidades, agilidad y destreza en los procedimientos, dedicación y, por qué no decirlo, pasión, así como flexibilidad de pensamiento y capacidad de adaptación a las necesidades de cada paciente y del equipo neuroquirúrgico(6). Es muy importante la mejora continua por parte del equipo de anestesiología, siempre actuar con base en las evidencias clínicas en beneficio y satisfacción del paciente, en la experiencia que se tenga y en las políticas institucionales del lugar en donde se desenvuelva profesionalmente ${ }^{(12)}$.

\section{REFERENCIAS}

1. Meng L, McDonagh DL, Berger MS, Gelb AW. Anesthesia for awake craniotomy: a how-to guide for the occasional practitioner. Can J Anaesth. 2017;64:517-529.

2. Ramírez-Segura EH. Anestesia para craneotomía con el paciente despierto: Técnica dormido-despierto-dormido. Rev Mex Anest. 2014;37:48-52.

3. Jaramillo MJ. Anestesia para craneotomía con el paciente despierto. Rev Mex Anest. 2009;32:S77-S78.

4. Abdulrauf SI, Urquiaga JF, Patel R, Albers JA, Sampat VB, Baumer $\mathrm{M}$, et al. Awake microvascular decompression for trigeminal neuralgia: concept and initial results. World Neurosurg. 2018;113: e309-e313.

5. Huncke K, Van de Wiele B, Fried I, Rubinstein EH. The asleep-awakeasleep anesthetic technique for intraoperative language mapping. Neurosurgery. 1998;42:1312-1316.

6. Flexman AM, Wang T, Meng L. Neuroanesthesia and outcomes: evidence, opinions, and speculations on clinically relevant topics. Curr Opin Anaesthesiol. 2019;32:539-545.

7. Klimek M, Verbrugge SJ, Roubos S, van der Most E, Vincent AJ, Klein J. Awake craniotomy for glioblastoma in a 9-year-old child. Anaesthesia. 2004;59:607-609.

8. Hedayat H, Felbaum DR, Reynolds JE, Janjua RM. Awake craniotomy for the treatment of a cortical pseudoaneurysm in a pregnant patient. Cureus. 2017;9:e1921.

9. Guevara-Pérez A, León-Álvarez E. Técnica dormido-despierto-dormido en paciente pediátrico: presentación de un caso. Anestesia en México. 2020;32:75-81.

10. León-Álvarez E. Anestesia en neurocirugía funcional en pediatría. Rev Mex Anest. 2010;33:122-124.

11. Garcia-Navarrete R, Terrazo-Lluch J, Marhx-Bracho A, León Alvárez E, Olvera González N, Alvárez-Mora B, et al. Awake craniotomy and brain mapping for brain tumor resection in pediatric patients. 2021. doi: 10.5772/intechopen.97101.

12. Eseonu CI, Rincon-Torroella J, ReFaey K, Quiñones-Hinojosa A. The cost of brain surgery: awake vs asleep craniotomy for perirolandic region tumors. Neurosurgery. 2017;81:307-314.
13. Meng L, Berger MS, Gelb AW. The potential benefits of awake craniotomy for brain tumor resection: an anesthesiologist's perspective. J Neurosurg Anesthesiol. 2015;27:310-317.

14. Gerritsen JKW, Klimek M, Dirven CMF, Hoop EO, Wagemakers M, Rutten GJM, et al. The SAFE-trial: Safe surgery for glioblastoma multiforme: Awake craniotomy versus surgery under general anesthesia. Study protocol for a multicenter prospective randomized controlled trial. Contemp Clin Trials. 2020;88:105876.

15. Sower VE. Great Ormond street hospital for children: Ferrari's formula one handovers and handovers from surgery to intensive care. The American Society for Quality. 2008.

16. Gernsback JE, Kolcun JPG, Starke RM, Ivan ME, Komotar RJ. Who needs sleep? An analysis of patient tolerance in awake craniotomy. World Neurosurg. 2018;118:e842-e848.

17. Kim SH, Choi SH. Anesthetic considerations for awake craniotomy. Anesth Pain Med (Seoul). 2020;15:269-274.

18. Marenco-Hillembrand L, Suarez-Meade P, Sabsevitz DS, Leone BJ, Chaichana KL. Awake craniotomy in a patient with previously diagnosed post-traumatic stress disorder. World Neurosurg. 2020;139:7-11.

19. Sitnikov AR, Grigoryan YA, Mishnyakova LP. Awake craniotomy without sedation in treatment of patients with lesional epilepsy. Surg Neurol Int. 2018;9:177.

20. Quintana-Schmidt C, Lladó-Carbo E, Cortés-Doñate VE, Grupo de trabajo de Neurooncología (GTNO), Sociedad Española de Neurofisiología Clínica (SENFC). Opciones de monitorización neurofisiológica en la resección de tumoraciones cerebrales. Documento de consenso entre el Grupo de Trabajo de Neurooncología de la Sociedad Española de Neurocirugía (SENEC) y la Sociedad Española de Neurofisiología Clínica (SENFC). Neurocirugía. 2018;29:25-38.

21. Hamacek de Freitas C, Santos-Oliveira CH. Consideracoes anestésicas para craniotomia em paciente acordado: relato de caso. Rev Bras Anestesiol. 2018;68:311-314.

22. Meziane M, Elkoundi A, Ahtil R, Guazaz M, Mustapha B, Haimeur C. Anaesthetic management for awake craniotomy in brain glioma resection: initial experience in Military Hospital Mohamed V of Rabat. Pan Afr Med J. 2017;27:156. 
23. Elbakry AE, Ibrahim E. Propofol-dexmedetomidine versus propofolremifentanil conscious sedation for awake craniotomy during epilepsy surgery. Minerva Anestesiol. 2017;83:1248-1254.

24. Soehle M, Wolf CF, Priston MJ, Neuloh G, Bien CG, Hoeft A, et al. Propofol pharmacodynamics and bispectral index during key moments of awake craniotomy. J Neurosurg Anesthesiol. 2018;30:32-38.
25. Cormack J, Karna S. Macroglossia during awake craniotomy: a near miss. J Neurosurg Anesthesiol. 2018;30:383-384.

26. Spena G, Roca E, Guerrini F, Panciani PP, Stanzani L, Salmaggi A et al. Risk factors for intraoperative stimulation-related seizures during awake surgery: an analysis of 109 consecutive patients. J Neurooncol. 2019;145:295-300. 